\title{
Nonlinear EGR and VGT Control with Integral Action for Diesel Engines
}

\author{
J. Wahlström* and L. Eriksson \\ Dept. of Electrical Engineering, Linköping University, 58183 Linköping - Sweden \\ e-mail: johan.erik.wahlstrom@gmail.com - larer@isy.liu.se \\ * Corresponding author
}

\begin{abstract}
Résumé - Régulation de Recirculation des Gaz d'Echappement (RGE) et de Turbine à Géométrie Variable (TGV) non linéaire avec action intégrée pour moteurs Diesel - Une conception de régulation non linéaire à variables multiples avec action intégrée est proposée et étudiée pour une régulation de la Recirculation des Gaz d'Echappement (RGE) et une Turbine à Géométrie Variable (TGV) au sein de moteurs Diesel à usage industriel. L'objectif principal de la régulation consiste à réguler le rapport oxygène / carburant et la fraction de RGE de tubulure d'admission, qui sont spécifiés au sein d'une boucle extérieure. Ceux-ci sont choisis en tant que variables de performance principales puisqu'ils sont fortement associés aux émissions. Une conception de régulation non linéaire existante basée sur une linéarisation en retour est étendue avec une action intégrée. En particulier, la méthode de conception de régulation utilise une fonction de Lyapunov, une régulation optimale inverse et une transformation de données d'entrée non linéaire. Des comparaisons entre des structures de régulation différentes sont réalisées par des simulations présentant les quatre points suivants. Premièrement, une action intégrée est nécessaire pour gérer les erreurs de modèle, de sorte que le régulateur puisse suivre les variables de performance spécifiées dans la boucle extérieure. Deuxièmement, la conception de régulation proposée gère les effets non linéaires au sein du moteur Diesel, ce qui conduit à des erreurs de régulation moindres par comparaison avec une structure de régulation au moyen de régulateurs PID. Troisièmement, il est important d'utiliser la transformation de données d'entrée et il est suffisant d'utiliser une structure de régulateur dotée de contrôleurs PID et d'une transformation de données d'entrée pour gérer les effets non linéaires. Quatrièmement, la conception de régulation proposée est sensible aux erreurs de modèle au cours de la transformation de données d'entrée tandis qu'une structure de régulation dotée de contrôleurs PID et d'une transformation d'entrée gère ces erreurs de modèle.
\end{abstract}

\footnotetext{
Abstract - Nonlinear EGR and VGT Control with Integral Action for Diesel Engines - A nonlinear multivariable control design with integral action is proposed and investigated for control of Exhaust Gas Recirculation (EGR) and Variable Geometry Turbine (VGT) in heavy duty Diesel engines. The main control goal is to regulate oxygen/fuel ratio and intake manifold EGR-fraction, and they are specified in an outer loop. These are chosen as main performance variables since they are strongly coupled to the emissions. An existing nonlinear control design based on feedback linearization is extended with integral action. In particular, the control design method utilizes a control Lyapunov function, inverse optimal control, and a nonlinear input transformation. Comparisons between different control structures are performed in simulations showing the following four points. Firstly, integral action is necessary to handle model errors so that the controller can track the performance variables specified in the outer loop. Secondly, the proposed control design handles the nonlinear effects in the
} 
Diesel engine that results in less control errors compared to a control structure with PID controllers. Thirdly, it is important to use the input transformation and it is sufficient to use a control structure with PID controllers and input transformation to handle the nonlinear effects. Fourthly, the proposed control design is sensitive to model errors in the input transformation while a control structure with PID controllers and input transformation handles these model errors.

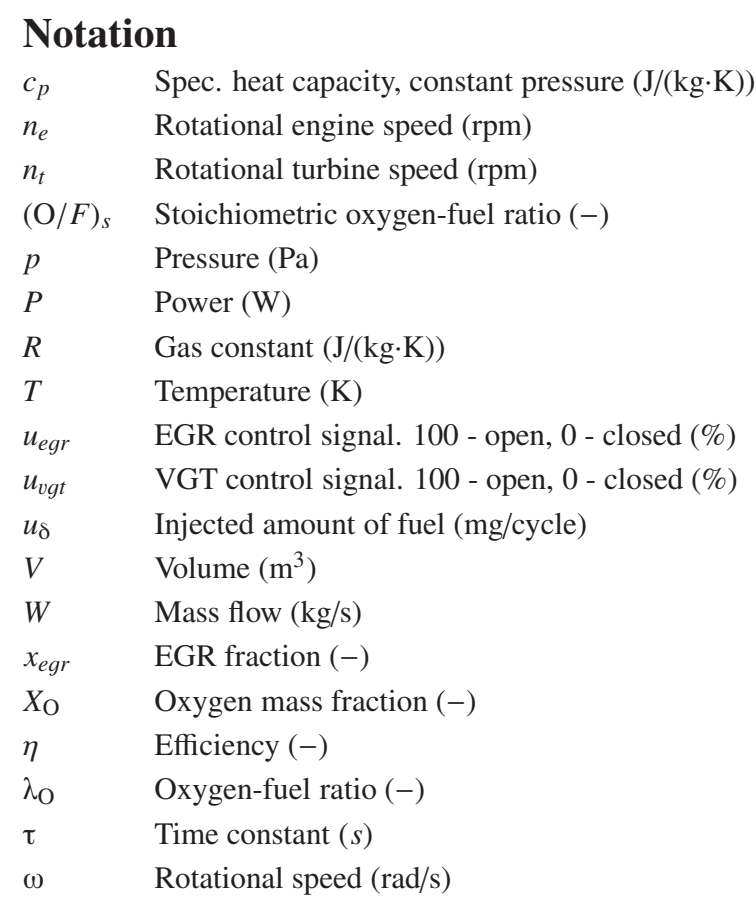

\section{Indices}

a Air

amb Ambient

c Compressor

e Exhaust

egr EGR

ei Engine cylinder in

em Exhaust manifold

eo Engine cylinder out

$f \quad$ Fuel

im Intake manifold

$m \quad$ Mechanical

$t \quad$ Turbine

vgt VGT

$\delta \quad$ Fuel injection

\section{INTRODUCTION}

Legislated emission limits for heavy duty trucks are constantly reduced. To fulfill the requirements, technologies like Exhaust Gas Recirculation (EGR) and Variable
Geometry Turbine (VGT) have been introduced. The primary emission reduction mechanisms utilized to control the emissions are that $\mathrm{NO}_{x}$ can be reduced by increasing the intake manifold EGR-fraction $x_{\text {egr }}$ and smoke can be reduced by increasing the air/fuel ratio [1]. Note that exhaust gases, present in the intake, also contain oxygen which makes it more suitable to define and use the oxygen/fuel ratio $\lambda_{\mathrm{O}}$ instead of the traditional air/fuel ratio. The main motive for this is that it is the oxygen contents that is crucial for smoke generation. Besides $\lambda_{\mathrm{O}}$ it is natural to use EGR-fraction $x_{e g r}$ as the other main performance variable, but one could also use the burned gas fraction instead of the EGR-fraction.

The oxygen/fuel ratio $\lambda_{\mathrm{O}}$ and EGR fraction $x_{e g r}$ depend in complicated ways on the EGR and VGT actuation. It is therefore necessary to have coordinated control of the EGR and VGT to reach the legislated emission limits in $\mathrm{NO}_{x}$ and smoke. Various approaches for coordinated control of the EGR and VGT for emission abatement have been published. Reference [2] presents a good overview of different control aspects of Diesel engines with EGR and VGT, and in [3] there is a comparison of some control approaches with different selections of performance variables. Other control approaches are described in [4-12].

A nonlinear multivariable control design is proposed in [13]. This design includes construction of a Lyapunov function, inverse optimal control, and a nonlinear input transformation which provides a control law that handles interactions and nonlinear properties in the system. The compressor mass flow $W_{c}$ and exhaust manifold pressure $p_{e m}$ are chosen as outputs, and therefore the set-points for $\lambda_{\mathrm{O}}$ and $x_{e g r}$ are transformed to set-points for $W_{c}$ and $p_{e m}$. This set-point transformation is based on a third-order model that describes the most important dynamics in the engine: the pressure dynamics in the intake and exhaust manifolds and the turbocharger dynamics. The third order nonlinear model captures the main system properties, such as non-minimum phase behaviors and sign reversals. If the control design is applied to a real engine, there will be control errors for $\lambda_{\mathrm{O}}$ and $x_{e g r}$ due to model errors in the third order model. In order to decrease these control errors, [14] proposes a control design that extends the one in [13] with integral action. This paper briefly reviews the design in [14], analyzes the robustness of this structure, and illustrates the sensitivity to model uncertainties in the input transformation by comparing the design in [14] to a control structure with PID 
controllers and a nonlinear input transformation. The main goal is to perform these comparisons for the performance variables $\lambda_{\mathrm{O}}$ and $x_{e g r}$ in operating points where the actuators are fully controllable. Further, the paper includes modeling, control design, tuning, and simulation.

\section{DIESEL ENGINE MODEL}

A model for a heavy duty Diesel engine is used in simulation for tuning and validation of the developed controller. This Diesel engine model is focused on the gas flows, (see Fig. 1), and it is a mean value model with eight states: intake and exhaust manifold pressures $\left(p_{i m}\right.$ and $\left.p_{e m}\right)$, oxygen mass fraction in the intake and exhaust manifold $\left(X_{\mathrm{Oim}}\right.$ and $\left.X_{\mathrm{Oem}}\right)$, turbocharger speed $\left(\omega_{t}\right)$, and three states $\left(\tilde{u}_{e g r 1}, \tilde{u}_{e g r 2}\right.$, and $\tilde{u}_{v g t}$ ) describing the actuator dynamics for the two control signals $\left(u_{\text {egr }}\right.$ and $\left.u_{v g t}\right)$. These states are collected in a state vector $x$ :

$$
x=\left[\begin{array}{cccc}
p_{i m} & p_{e m} & X_{\mathrm{O} i m} & X_{\mathrm{O} e m} \\
\omega_{t} & \tilde{u}_{e g r 1} & \tilde{u}_{e g r 2} & \left.\tilde{u}_{v g t}\right]^{T}
\end{array}\right.
$$

The resulting model is expressed in state space form as:

$$
\dot{x}=f\left(x, u, n_{e}, u_{\delta}\right)
$$

where the engine speed $n_{e}$ and the mass of injected fuel $u_{\mathrm{\delta}}$ are considered as disturbances, and $u$ is the control input vector $u=\left[\begin{array}{ll}u_{e g r} & u_{v g t}\end{array}\right]^{T}$ which contains EGR-valve position $u_{e g r}$ and VGT actuator position $u_{v g t}$. Fuel control is important but it is not the focus in this paper. This paper focuses on EGR and VGT control.

A detailed description and derivation of the model together with a model tuning and a validation against test cell measurements is given in [15]. The derivatives of the engine state variables are given by Equations (1), and the

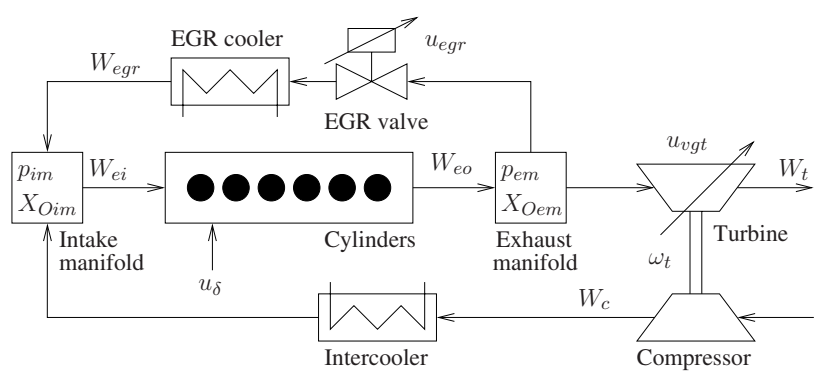

Figure 1

Sketch of the Diesel engine model used for simulation, control design, and tuning. It has five states related to the engine $\left(p_{i m}\right.$, $p_{e m}, X_{\mathrm{Oim}}, X_{\mathrm{Oem}}$, and $\left.\omega_{t}\right)$ and three for actuator dynamics. oxygen concentration in the exhaust gas is calculated in (2). Further, the main performance variables are defined by (3).

$$
\begin{aligned}
\frac{d}{d t} p_{e m}= & f_{1}(x, u), \quad \frac{d}{d t} \omega_{t}=f_{2}(x, u) \\
\frac{d}{d t} p_{i m}= & \frac{R_{a} T_{i m}}{V_{i m}}\left(W_{c}+W_{e g r}-W_{e i}\right) \\
\frac{d}{d t} X_{\mathrm{O} i m}= & \frac{R_{a} T_{i m}}{p_{\text {im }} V_{i m}}\left(\left(X_{\mathrm{O} e m}-X_{\mathrm{O} i m}\right) W_{e g r}\right. \\
& \left.+\left(X_{\mathrm{O} c}-X_{\mathrm{O} i m}\right) W_{c}\right) \\
\frac{d}{d t} X_{\mathrm{O} e m}= & \frac{R_{e} T_{e m}}{p_{e m} V_{e m}}\left(X_{\mathrm{O} e}-X_{\mathrm{O} e m}\right)\left(W_{f}+W_{e i}\right) \\
X_{\mathrm{O} e}= & \frac{W_{e i} X_{\mathrm{O} i m}-W_{f}(\mathrm{O} / F)_{s}}{W_{f}+W_{e i}} \\
x_{e g r}= & \frac{W_{e g r}}{W_{c}+W_{e g r}}, \quad \lambda_{\mathrm{O}}=\frac{W_{e i} X_{\mathrm{O} i m}}{W_{f}(\mathrm{O} / F)_{s}}
\end{aligned}
$$

\section{ROBUST NONLINEAR CONTROL}

The control design method used is based on a nonlinear multivariable method proposed in [16] and [17]. It includes construction of a Control Lyapunov Function (CLF) and inverse optimal control that guarantees robustness of optimal controllers. The control design method is briefly reviewed below.

Consider the system:

$$
\begin{aligned}
& \dot{x}=f(x)+g(x) u \\
& y=h(x)+j(x) u, \quad x \in \mathbb{R}^{n}, \quad u, y \in \mathbb{R}^{m}
\end{aligned}
$$

where $u=0$ render the equilibrium point $x=0$. As mentioned above, the control design method includes construction of a CLF $V(x)$ that is defined as follows.

Definition 1 (Control Lyapunov Function) A radially unbounded, positive definite, smooth scalar function $V(x)$ is called a Control Lyapunov Function (CLF) for (4) if there exists a $u$ such that $\dot{V}(x)=L_{f} V(x)+L_{g} V(x) u<0$ for all $x \neq 0$. The notation $L_{q} V(x)$ denotes the Lie derivate of $V(x)$ along the vector field $q(x)$.

The control design method also includes inverse optimal control, where the goal is to first design a control law and then determine which cost function it minimizes. In order to obtain a simple relation between the control law and the cost function, the cost function is chosen quadratic in $u$ according to:

$$
\int_{0}^{\infty} l(x)+u^{T} R(x) u d t, \quad l(x)>0, \quad R(x)>0
$$


In standard optimal control [18], the control law that minimizes (5) is found by solving the Hamilton-Jacobi-Bellman equation:

$$
0=\min _{u}\left[l(x)+u^{T} R(x) u+L_{f} V(x)+L_{g} V(x) u\right]
$$

where $V(x)$ is a CLF. Equation (6) has the solution:

$$
u(x)=-\frac{1}{2} R^{-1}\left(L_{g} V\right)^{T}(x)
$$

Consequently, the goal is to first design $V(x)$ and the matrix $R(x)$, and then determine $l(x)$ in order to see which cost function the control law (7) minimizes. The function $l(x)$ is found by solving Equation (6) for $l(x)$, with the control law (7) inserted, yielding

$$
l(x)=\frac{1}{4} L_{g} V(x) R^{-1}\left(L_{g} V\right)^{T}(x)-L_{f} V(x)
$$

The optimal control law (7) guarantees stability and robustness properties. In particular, if

$$
l(x)>0
$$

and $R(x)$ is diagonal then the control law (7) gives asymptotic stability and it is robust to static input uncertainties and has $(1 / 2, \infty)$ gain margin [16].

\section{CONTROL DESIGN WITH INTEGRAL ACTION}

A control design without integral action of a Diesel engine using the method in Section 2 is proposed in [13]. This gives an inner loop that handles nonlinearities and decouples the system. However, as will be shown in Section 5, integral action is necessary to handle model errors so that the controller can track the performance variables $\lambda_{\mathrm{O}}$ and $x_{\text {egr }}$ specified in an outer loop. Therefore, the proposed design is extended with a single integral action, resulting in the proposed closed-loop system with a single integral action shown in Figure 2. Reference [6] proposes also a single integral action, however the integral action here is used in other way compared to [6] in order to track the performance variables $\lambda_{\mathrm{O}}$ and $x_{\text {egr }}$.

\subsection{Control Design Model}

To get a simple control law, the eighth order model in Section 1 is simplified to a model with three states: $p_{i m}, p_{e m}$, and the compressor power $P_{c}$. This model is based on the control design model developed in [13].

$$
\begin{gathered}
\dot{p}_{i m}=k_{i m}\left(W_{c}+u_{1}-k_{e} p_{i m}\right) \\
\dot{p}_{e m}=k_{e m}\left(k_{e} p_{\text {im }}-u_{1}-u_{2}+W_{f}\right) \\
\dot{P}_{c}=\frac{1}{\tau}\left(\eta_{m} P_{t}-P_{c}\right) \\
W_{c}=\frac{\eta_{c} P_{c}}{T_{a m b} c_{p a}\left(\left(p_{i m} / p_{a m b}\right)^{\left.\mu_{a}-1\right)}\right.} \\
P_{t}=\eta_{t} c_{p e} T_{e m}\left(1-\left(p_{a m b} / p_{e m}\right)^{\mu_{e}}\right) u_{2}
\end{gathered}
$$

where $u_{1}=W_{\text {egr }}$ is the EGR flow and $u_{2}=W_{t}$ is the turbine flow, where $u_{1}$ and $u_{2}$ are treated as control inputs. The values for $u_{e g r}$ and $u_{v g t}$ are then obtained by using a nonlinear input transformation according to Figure 2. This input transformation is an inversion of position to flow models where the actuator dynamics is neglected, see [19] for more details. The variables $k_{e m}=k_{e m}\left(T_{e m}\right), W_{f}=W_{f}\left(u_{\delta}, n_{e}\right)$, and $k_{e}=k_{e}\left(n_{e}\right)$ are treated as external slowly varying signals and $k_{i m}, \tau, \eta_{m}, \eta_{c}, T_{a m b}, c_{p a}, p_{a m b}, \mu_{a}, \eta_{t}, c_{p e}$, and $\mu_{e}$ are constants. This model is the same as the control design model developed in [13] if $c_{p a}=c_{p e}$ and $\mu_{a}=\mu_{e}$. A linearization of the model (10) is performed in several operating points covering the complete operating region showing that these linearized models are unstable for all the considered operating points.

\subsection{Outputs and Set-Points}

The design objective is to regulate $\lambda_{\mathrm{O}}$ and $x_{\text {egr }}$ to their setpoints $\lambda_{\mathrm{O}}^{s}$ and $x_{\text {egr }}^{s}$. However, $\lambda_{\mathrm{O}}$ can not be calculated from the control design model in Section 3.1. Further, $p_{e m}$ has to

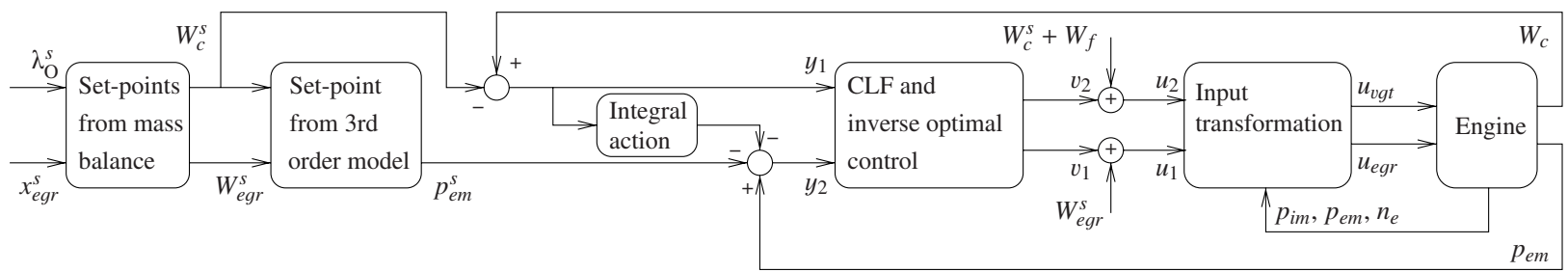

Figure 2

Block diagram of the closed-loop system, showing; set-points calculations, integral action on the flow $W_{c}$, and nonlinear control of the flows $u_{1}=W_{e g r}$ and $u_{2}=W_{t}$. The nonlinear input transformation is an inversion of position to flow models where the actuator dynamics is neglected, see [19] for more details. 
be chosen as one output in order to get stable zero dynamics [13]. Therefore, the following outputs are chosen:

$$
y_{1}=W_{c}-W_{c}^{s}, \quad y_{2}=p_{e m}-p_{e m}^{s}
$$

The set-points $\lambda_{\mathrm{O}}^{s}$ and $x_{e g r}^{s}$ are transformed to the set-points $W_{c}^{s}$ and $p_{e m}^{s}$ in two steps. Firstly, the equilibria for $W_{c}$ and $W_{\text {egr }}$ of the mass balances $(1 \mathrm{~b}-1 \mathrm{~d})$ are calculated from $\lambda_{\mathrm{O}}^{s}$ and $x_{\text {egr }}^{s}$ :

$$
\begin{aligned}
W_{c}^{s} & =\frac{W_{f}}{2 X_{\mathrm{O} c}}\left(\beta+\sqrt{\beta \frac{1}{2}+4 \lambda_{\mathrm{O}}^{s}(\mathrm{O} / F)_{s}\left(1-x_{\text {egr }}^{s}\right) X_{\mathrm{O} c}}\right) \\
W_{e g r}^{s} & =\frac{x_{e g r}^{s}}{1-x_{\text {egr }}^{s}} W_{c}^{s}
\end{aligned}
$$

where $\beta=\left(\lambda_{\mathrm{O}}^{s}(\mathrm{O} / F)_{s}-X_{\mathrm{O} c}\right)\left(1-x_{\text {egr }}^{s}\right)+(\mathrm{O} / F)_{s} x_{\text {egr }}^{s}, X_{\mathrm{O} c}$ is the constant oxygen concentration in air passing the compressor, and $(\mathrm{O} / F)_{s}$ is the stoichiometric relation between oxygen and fuel masses. Secondly, the equilibria for $p_{i m}$ and $p_{\text {em }}$ of the third-order model in Section 3.1 are calculated from $W_{c}^{s}$ and $W_{e g r}^{s}$ :

$$
\begin{aligned}
& p_{\text {im }}^{s}=\frac{W_{c}^{s}+W_{e g r}^{s}}{k_{e}} \\
& p_{e m}^{s}=p_{a m b}\left(1-\frac{c_{p a}\left(\left(\frac{p_{i m}^{s}}{p_{a m b}}\right)^{\mu_{a}}-1\right) T_{a m b} W_{c}^{s}}{c_{p e} \eta_{c m t} T_{e m}\left(W_{c}^{s}+W_{f}\right)}\right)^{-\frac{1}{\mu_{e}}}
\end{aligned}
$$

where $\eta_{c m t}=\eta_{c} \eta_{m} \eta_{t}$.

\subsection{Integral Action}

The set-points in (12) are given by the equilibria (14) for the third order model, which is a simplification of the real system. When the control design is applied to the real system the model errors will propagate to stationary errors in $\lambda_{\mathrm{O}}$ and $x_{\text {egr }}$ (this will be illustrated in Fig. 4). To decrease these stationary errors, the following approximate integral action is used:

$$
\frac{d i}{d t}=-\delta i-K\left(W_{c}-W_{c}^{s}\right)=-\delta i-K y_{1}
$$

where $\delta$ is small and positive to ensure stable zero dynamics. The choice of $y_{1}$ as input to the integral action ensures that the set-point $W_{c}^{s}$ is achieved. Integral action can not be performed on $\lambda_{O}$ since $\lambda_{O}$ can not be calculated from the control design model. Further, if integral action is performed on $x_{\text {egr }}$, stable zero dynamics can not be guaranteed.

The next step is then to ensure that $W_{e g r}^{s}$ is achieved and this is done by feeding the state $i$ into $y_{2}$ yielding the outputs:

$$
y_{1}=W_{c}-W_{c}^{s}, \quad y_{2}=p_{e m}-p_{e m}^{s}-d_{1} i / d_{2}
$$

The motive for feeding the state $i$ into $y_{2}$ is as follows. The integral action (15) and the outputs (16) lead to that $y_{1}=$ $y_{2}=0$ in stationary points, leading to $v_{1}=v_{2}=0$ according to (26) yielding $u_{1}=W_{\text {egr }}^{s}$ according to Figure 2, i.e. $W_{\text {egr }}^{s}$ is achieved. The nonlinear gain $d_{1} / d_{2}$ in (16) where:

$$
\begin{aligned}
& d_{1}=\left[\mu_{a} W_{c}^{s}\left(W_{c}^{s}+W_{f}\right)\left(W_{c}^{s}+W_{e g r}^{s}\right)^{\mu_{a}-1}\right. \\
& \left.+\left(\left(W_{c}^{s}+W_{e g r}^{s}\right)^{\mu_{a}}-k_{e}^{\mu_{a}} p_{a m b}{ }^{\mu_{a}}\right) W_{f}\right] c_{p a}\left(p_{e m}^{s}\right)^{\mu_{e}+1} T_{a m b} \\
& d_{2}=c_{p e} \eta_{c m t} k_{e}^{\mu_{a}} \mu_{e} p_{a m b}{ }^{\mu_{a}+\mu_{e}} T_{e m}\left(W_{c}^{s}+W_{f}\right)^{2}
\end{aligned}
$$

is developed by investigating $\frac{d}{d W_{c}^{s}} p_{e m}^{s}\left(W_{c}^{s}\right)$, see [14] for more details.

\subsection{Feedback Linearization}

The first step in the control design method is to construct a CLF $V(x)$, which is done using feedback linearization. For the fourth order model (10) and (15), and the outputs (16), the relative degrees become $r_{1}=1$ and $r_{2}=1$. Consequently, $\dot{y}$ can be formulated as:

$$
\dot{y}=G(y, z) u+F(y, z)
$$

where $y=\left[\begin{array}{ll}y_{1} & y_{2}\end{array}\right]^{T}, u=\left[u_{1} u_{2}\right]^{T}, z=\left[p_{i m}, i\right]^{T}$, the matrix

$$
G(y, z)=\left[\begin{array}{cc}
-a & b \\
-k_{e m} & -k_{e m}
\end{array}\right]
$$

where

$$
\begin{aligned}
& a=\frac{k_{i m} \mu_{a}\left(\frac{p_{i m}}{p_{a m b}}\right)^{\mu_{a}-1} W_{c}}{p_{a m b}\left(\left(\frac{p_{i m}}{p_{a m b}}\right)^{\mu_{a}}-1\right)} \\
& b=\frac{c_{p e} \eta_{c m t}\left(1-\left(\frac{p_{a m b}}{p_{e m}}\right)^{\mu_{e}}\right) T_{e m}}{c_{p a}\left(\left(\frac{p_{\text {im }}}{p_{a m b}}\right)^{\mu_{a}}-1\right) \tau T_{a m b}}
\end{aligned}
$$

and where

$$
F(y, z)=\left[\begin{array}{c}
a k_{e} p_{i m}-a W_{c}-\frac{W_{c}}{\tau} \\
\frac{d_{1}\left(\delta i+K\left(W_{c}-W_{c}^{s}\right)\right)}{d_{2}}+k_{e m}\left(k_{e} p_{i m}+W_{f}\right)
\end{array}\right]
$$

In order to use feedback linearization, it must be shown that the matrix $G(y, z)$ above is invertible, which is done in the following way. Assuming that $p_{e m}>p_{a m b}$ and $u_{2}>0$, which are reasonable assumptions for a physical engine with a turbocharger, the set $\Omega=\left\{\left(p_{i m}, P_{c}\right): p_{i m}>p_{a m b}, P_{c}>0\right\}$ is invariant, i.e. every trajectory starting in $\Omega$ stays in $\Omega$ for all $t$. This can be shown in the following way. Using the assumptions above, (11b) leads to that $P_{t}>0$. Then, by choosing a starting point $P_{c}(t=0)>0$ and $p_{\text {im }}(t=0)>p_{\text {amb }}$ for a trajectory, (10c) gives that $P_{c}>0$ for all $t \geq 0$ since (10c) is a linear first order system with $P_{t}$ as input. Further, by using the starting point in the trajectory 
above and (11a), $W_{c} \rightarrow+\infty$ when $p_{i m} \rightarrow p_{a m b}$, leading to that $\dot{p}_{\text {im }} \rightarrow+\infty$ when $p_{\text {im }} \rightarrow p_{\text {amb }}$, i.e. $p_{\text {im }}>p_{\text {amb }}$ for all $t \geq 0$, which proves that the set $\Omega$ above is invariant. Using this result, $a, b>0$ and $G(y, z)$ is invertible.

Then by applying the nonlinear cancellation:

$$
u=G^{-1}(y, z)(-\alpha y-F(y, z)+w)
$$

and the change of coordinates $x \rightarrow[y, z]$, the system (10) and (15) is transformed into the system:

$$
\begin{aligned}
& \dot{y}=-\alpha y+w \\
& \dot{z}=f_{0}(y, z)+g_{0}(y, z) w
\end{aligned}
$$

where $w$ is the new input and $\alpha$ is a positive scalar constant.

\subsection{Stability of the Zero Dynamics}

When feedback linearization is used it is necessary to investigate the stability of the zero dynamics, that is defined by

$$
\dot{z}=f_{0}(0, z)
$$

For Equation (22) the zero dynamics becomes:

$$
\begin{aligned}
\frac{d p_{i m}}{d t} & =\frac{q_{1}\left(p_{\text {im }}\right)}{q_{2}\left(p_{i m}, i\right)}\left(p_{i m}^{\mu_{a}}-q_{3}(i)\right) \\
\frac{d i}{d t} & =-\delta i
\end{aligned}
$$

where

$$
\begin{aligned}
q_{1}\left(p_{i m}\right)= & c_{p a} k_{i m} p_{i m} T_{a m b} W_{c}^{s} \\
q_{2}\left(p_{i m}, i\right)= & -c_{p a} k_{i m} \mu_{a} p_{i m}^{\mu_{a}} T_{a m b} \tau W_{c}^{s} \\
& -c_{p e} \eta_{c m t} p_{a m b}{ }^{\mu_{a}}\left(1-\left(\frac{p_{a m b}}{\frac{d_{1} i}{d_{2}}+p_{e m}^{s}}\right)^{\mu_{e}}\right) p_{i m} T_{e m} \\
q_{3}(i)= & \frac{p_{a m b}{ }^{\mu_{a}}}{c_{p a} k_{e m} T_{a m b} W_{c}^{s}}\left(\left(\frac{d_{1} \delta i}{d_{2}}+k_{e m}\left(W_{c}^{s}+W_{f}\right)\right) .\right. \\
& \left.\left(1-\left(\frac{p_{a m b}}{\frac{d_{1} i}{d_{2}}+p_{e m}^{s}}\right)^{\mu_{e}}\right) c_{p e} \eta_{c m t} T_{e m}+c_{p a} k_{e m} T_{a m b} W_{c}^{s}\right)
\end{aligned}
$$

To analyze the stability of the zero dynamics $(23,24)$, the Lyapunov function

$$
V_{z}=\frac{1}{2} c_{z 1} z_{1}^{2}+\frac{1}{2} c_{i} i^{2}
$$

is used where $z_{1}=p_{i m}^{\mu_{a}}-q_{3}(i)$. The zero dynamics $(23,24)$ is asymptotically stable if

$$
\begin{aligned}
\dot{V}_{z}= & c_{z 1} z_{1}^{2}\left(\mu_{a} p_{i m}^{\mu_{a}-1} \frac{q_{1}}{q_{2}}+\frac{\delta c_{z 1}\left(q_{3}^{\prime}(i)\right)^{2}}{4 c_{i}}\right) \\
& -c_{i} \delta\left(i-\frac{c_{z 1}}{2 c_{i}} q_{3}^{\prime}(i) z_{1}\right)^{2}<0
\end{aligned}
$$

for all $\left[z_{1}, i\right] \neq 0$ which is true if $\delta>0$ and

$$
\frac{c_{z 1}}{c_{i}}<-\frac{4}{\delta\left(q_{3}^{\prime}(i)\right)^{2}} \mu_{a} p_{i m}^{\mu_{a}-1} \frac{q_{1}}{q_{2}}
$$

This is possible if $q_{1} / q_{2}<0$, which is true if

$$
d_{1} i / d_{2}+p_{e m}^{s} \geq p_{a m b} .
$$

This relation is achieved by implementing the integral action (15) in discrete form with anti-windup according to:

$$
\begin{aligned}
& \epsilon_{n}=i_{n-1}-T_{s}\left(\delta i_{n-1}+K y_{1}\right) \\
& i_{n}= \begin{cases}\epsilon_{n}, & \text { if } \epsilon_{n} \geq i_{\text {min }} \\
i_{\text {min }}, & \text { if } \epsilon_{n}<i_{\text {min }}\end{cases}
\end{aligned}
$$

where $T_{s}$ is the sample time and

$$
i_{\text {min }}=-\frac{d_{2}}{d_{1}}\left(p_{e m}^{s}-p_{a m b}\right)
$$

\subsection{Construction of a CLF}

In order to obtain a simple control law, a quadratic Lyapunov function

$$
V=c_{1} y_{1}^{2}+c_{2} y_{2}^{2}
$$

is constructed, which is a CLF for the system (21), since

$$
\dot{V}=-2 c_{1} \alpha y_{1}^{2}-2 c_{2} \alpha y_{2}^{2}<0
$$

for $w=0$ and all $y \neq 0$.

\subsection{Control Law}

Using the CLF (25), the control law (7) can be applied to the system (21). However, this control law will contain the nonlinear cancellation (20) that is sensitive to model errors. Therefore, this cancellation is avoided by applying the control law (7) to the system (17) instead without using the nonlinear cancellation (20). The same Lyapunov function $V$ is used and since $V$ is a CLF for the system (21), it is a CLF for (17) which follows from Definition 1, see also [13] and [16].

To apply the control law (7), the control inputs are centered at their set-point values $v_{1}=u_{1}-W_{\text {egr }}^{s}$ and $v_{2}=u_{2}-W_{c}^{s}-W_{f}$. This leads to that $v=0$ renders the equilibrium point $y=0$.

A simple control law for the system (17) is achieved by choosing $R^{-1}=\operatorname{diag}\left\{\gamma_{1}, \gamma_{2}\right\}$, yielding

$$
\left[\begin{array}{l}
v_{1} \\
v_{2}
\end{array}\right]=-\frac{1}{2} R^{-1}\left(L_{G} V\right)^{T}=\left[\begin{array}{c}
\gamma_{1}\left(c_{1} a y_{1}+c_{2} k_{e m} y_{2}\right) \\
\gamma_{2}\left(-c_{1} b y_{1}+c_{2} k_{e m} y_{2}\right)
\end{array}\right]
$$

The last step is to find $l(x)$ and check the criterion (9). This is done in Proposition 1 in Appendix and the result is that 
there exist no $\gamma_{1}, \gamma_{2}, c_{1}, c_{2}$, and $K$ such that $l(x)>0$ for all $x$ and therefore there is no guarantee that the control law (26) gives a globally robust system according to Section 2. An analysis of $\dot{V}(x)$ also shows that there exist no $\gamma_{1}, \gamma_{2}, c_{1}, c_{2}$, and $K$ such that $\dot{V}(x)<0$ for all $x$ and therefore there is no guarantee that the control law (26) gives a globally asymptotically stable system. In Proposition 2 in Appendix, $l(x)$ and $\dot{V}(x)$ are also analyzed for the control design without integral action proposed in [13] showing that this design does neither guarantee a globally robust nor an asymptotically stable system for all $x$. However, it is shown in [13] that there exists a constant $c$ such that the design without integral action guarantees a locally asymptotically stable and robust system in the region $L=\{x: V(x) \leq c\}$ by selecting $\gamma_{1}$ and $\gamma_{2}$ sufficiently large.

Even without a control law that gives a globally asymptotically stable and robust system, there is a possibility that the control law (26) handles interactions and nonlinear properties in the system since the control law is model based. Therefore, the control performance of the proposed control structure is investigated in the following sections.

\section{AUTOMATIC OFFLINE CONTROLLER TUNING}

In the proposed control design there are five tuning parameters: $\gamma_{1}, \gamma_{2}, c_{1}, c_{2}$, and $K$. The tuning objectives are to minimize the error between $\lambda_{\mathrm{O}}$ and $\lambda_{\mathrm{O}}^{s}$, minimize the error between $x_{e g r}$ and $x_{e q r}^{s}$, and to achieve the inequality (9) for as many $x$ as possible. However, it is difficult to achieve these objectives by manual tuning, especially the last objective. Therefore a method for automatic offline tuning of the parameters is developed. It is based on the method in [20] but with a modified cost function.

\section{Cost Function for Tuning}

The automatic tuning method is obtained by formulating a nonlinear least squares problem:

$$
\min V(\theta) \text { s.t. } \theta>0
$$

where $\theta$ are the tuning parameters

$$
\theta=\left[\gamma_{1}, \gamma_{2}, c_{1}, c_{2}, K\right]^{T}
$$

The cost function $V(\theta)$ is calculated as:

$$
\begin{aligned}
V(\theta)= & \sum_{i=1}^{N} \gamma_{\lambda_{\mathrm{O}}}\left(\frac{e_{\lambda_{\mathrm{O}}}\left(t_{i}\right)}{\lambda_{\mathrm{ONorm}}}\right)^{2}+\gamma_{\text {egr }}\left(\frac{e_{\text {xegr }}\left(t_{i}\right)}{x_{\text {egrNorm }}}\right)^{2} \\
& +\left(\frac{u_{\text {egr }}\left(t_{i}\right)-u_{\text {egr }}\left(t_{i-1}\right)}{u_{\text {egrNorm }}}\right)^{2}+\left(\frac{u_{\text {vgt }}\left(t_{i}\right)-u_{\text {vgt }}\left(t_{i-1}\right)}{u_{\text {vgtNorm }}}\right)^{2} \\
& +\gamma_{l}\left(\frac{\min \left(l\left(x\left(t_{i}\right)\right), 0\right)}{l_{\text {Norm }}}\right)^{2}
\end{aligned}
$$

where $t_{i}$ is the time at sample number $i$. The motives for the different terms in the cost function are:

- Term 1: Minimizes $\lambda_{\mathrm{O}}$ error $\left(e_{\lambda_{\mathrm{O}}}=\lambda_{\mathrm{O}}^{s}-\lambda_{\mathrm{O}}\right)$ where a minimization of positive $\lambda_{\mathrm{O}}$ errors leads to less emissions and a minimization of negative $\lambda_{\mathrm{O}}$ errors leads to less pumping work [15]. Note that in [20] only positive $\lambda_{O}$ errors are minimized since negative $\lambda_{O}$ errors are very large when the actuators are saturated. However, here the main goal is to consider operating points where the actuators are fully controllable and for these operating points it is convenient to also minimize negative $\lambda_{\mathrm{O}}$ errors.

- Term 2: Minimizes EGR error $\left(e_{x e g r}=x_{e g r}^{s}-x_{e g r}\right)$.

- Term 3 and 4: These terms are coupled to the general issue of avoiding actuator stress, e.g. oscillatory behavior. The terms have equal weight.

- Term 5: If the inequality (9) is not satisfied, this term minimizes $l(x)^{2}$ using a high penalty, $\gamma_{l}=100$. Consequently, this term maximizes the number of operating points where the control law gives a robust system. However, this does not guarantee that the inequality (9) is satisfied for all operating points. For example, if the tuning method is applied to the transient cycles defined by Table $1, l(x)$ becomes negative in $31 \%$ of the total number samples.

As seen in Equation (28) all the terms are normalized to get the same order of magnitude for the five terms. The weighting factors $\gamma_{\lambda_{\mathrm{O}}}$ and $\gamma_{\text {egr }}$ are set to 3 and 1 , and the

\section{TABLE 1}

Table 1 defines 4 transient cycles that consist of steps between 13 different operating points. All cycles spend $10 \mathrm{~s}$ in each point. These cycles are used for tuning, and they cover a large operating region and capture important system properties such as nonlinear effects and sign reversals. The values for $\lambda_{\mathrm{O}}^{s}$ and $x_{\text {egr }}^{s}$ are obtained by calculating the stationary points of the eighth order model for the 13 points below. The operating points $O P_{1}$ and $O P_{2}$ are not used due to that $p_{e m}<p_{i m}$ in these operating points that might result in back-flow in the EGR-valve in a real engine. Instead, the operating point 7 is used where the EGR-valve is closed. The operating points $\mathrm{OP}_{3}$ and $O \mathrm{P}_{4}$ are not used due to that the turbocharger speed is too high in these points.

\begin{tabular}{c|c||c|c|c|c}
\hline$u_{\delta}(\mathrm{mg} /$ cycle $)$ & $u_{\text {egr }}(\%)$ & \multicolumn{5}{c}{ Operating points } \\
\hline \hline \multirow{3}{*}{230} & 40 & 6 & $O P_{1}$ & $O P_{3}$ & 9 \\
\cline { 2 - 6 } & 10 & 5 & $O P_{2}$ & $O P_{4}$ & 8 \\
\cline { 2 - 6 } & 0 & & 7 & & \\
\hline \multirow{2}{*}{60} & 40 & 4 & 3 & 11 & 10 \\
\cline { 2 - 6 } & 10 & 1 & 2 & 12 & 13 \\
\hline \hline & $u_{v g t}(\%)$ & 30 & 60 & 30 & 60 \\
\cline { 2 - 6 } & $n_{e}(\mathrm{rpm})$ & 1000 & 2000 \\
\hline \hline & Operating points used in each cycle \\
Cycle 1 & $1 \rightarrow 2 \rightarrow 3 \rightarrow 4 \rightarrow 1$ \\
Cycle 2 & $5 \rightarrow 6 \rightarrow 7 \rightarrow 5$ \\
Cycle 3 & $8 \rightarrow 9 \rightarrow 8$ \\
Cycle 4 & $10 \rightarrow 11 \rightarrow 12 \rightarrow 13 \rightarrow 10$ \\
\hline
\end{tabular}




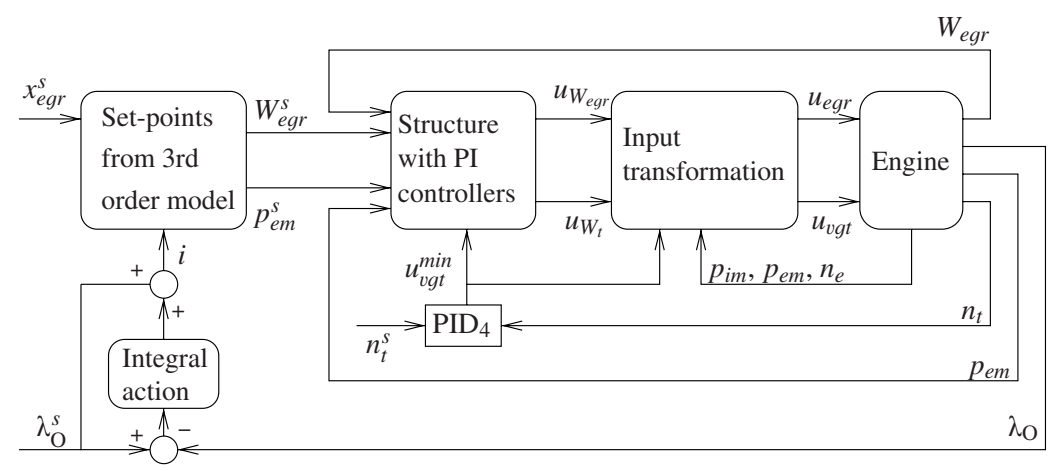

Figure 3

A control design with an integral action on the oxygen/fuel ratio $\lambda_{\mathrm{O}}$, set-points calculations, a structure with PI controllers, and an input transformation that is an inversion of position to flow models.

constant $\delta$ in $(15)$ is set to $10^{-6}$. To solve the optimization problem above, the solver in [20] is used.

\section{CONTROLLER EVALUATION}

The performance of the control system in Figure 2 is evaluated by comparing four different control systems:

- CLF with integral action: the control system in Figure 2 where the controller tuning method in Section 4 is applied to the transient cycles defined by Table 1 . These transients consist of steps in $\lambda_{\mathrm{O}}^{s}$ and $x_{\text {egr }}^{s}$ between 13 different operating points.

- CLF without integral action: same as above, but $K$ is set to zero and removed from $\theta$ in (27). Simulations show that there are negligible differences in control performance between "CLF without integral action" and the control design in [13]. In particular, these two controllers becomes equal if $c_{p a}=c_{p e}$ and $\mu_{a}=\mu_{e}$ in $(18,19)$ and if $c_{3}=0$ in [13].

- PID: the control structure with PID controllers and automatic tuning method proposed in [21]. It has the following control structure:

$$
\begin{aligned}
& u_{\text {egr }}\left(t_{i}\right)= \begin{cases}\min \left(-\mathrm{PI}_{1}\left(e_{\lambda_{\mathrm{O}}}\right),\right. & \text { if } u_{v g t}\left(t_{i-1}\right)=100 \\
\left.\mathrm{PI}_{2}\left(e_{x e g r}\right)\right), & \text { else } \\
-\mathrm{PI}_{1}\left(e_{\lambda_{\mathrm{O}}}\right), & \end{cases} \\
& u_{v g t}\left(t_{i}\right)=\left\{\begin{array}{l}
100, \quad \text { if }\left(u_{v g t}\left(t_{i-1}\right)=100\right) \&\left(e_{x e g r}<0.01\right) \\
\max \left(-\mathrm{PI}_{3}\left(e_{\text {xegr }}\right),\right. \\
\left.-\mathrm{PID}_{4}\left(e_{n t}\right)\right), \quad \text { else }
\end{array}\right. \\
& e_{\lambda_{\mathrm{O}}}=\lambda_{\mathrm{O}}^{s}-\lambda_{\mathrm{O}}, \quad e_{x e g r}=x_{e g r}^{s}-x_{e g r}, \quad e_{n t}=n_{t}^{s}-n_{t}
\end{aligned}
$$

- PID and input transformation: the control structure with PID controllers and a nonlinear input transformation in Figure 3 proposed in [19]. The input transformation is the same input transformation as in Figure 2. The block
"Structure with PI controllers"' in Figure 3 consists of the following equations:

$$
\begin{aligned}
& u_{W_{e g r}}\left(t_{i}\right)=\left\{\begin{array}{l}
W_{e g r}^{\max }, \\
\text { if }\left(u_{W_{e g r}}\left(t_{i-1}\right)=W_{\text {egr }}^{\max }\right) \text { and } \\
\left(e_{W_{\text {egr }}}>-5 \cdot 10^{-3}\right) \\
\mathrm{PI}_{1}\left(W_{\text {egr }}^{s}, W_{\text {egr }}\right), \text { else }
\end{array}\right. \\
& u_{W_{t}}\left(t_{i}\right)=\left\{\begin{array}{l}
\min \left(-\mathrm{PI}_{2}\left(p_{\text {em }}^{s}, p_{\text {em }}\right),-\mathrm{PI}_{3}\left(W_{\text {egr }}^{s}, W_{\text {egr }}\right)\right) \\
\text { if } u_{W_{\text {egr }}}\left(t_{i-1}\right)=W_{\text {egr }}^{\max } \\
-\mathrm{PI}_{2}\left(p_{\text {em }}^{s}, p_{\text {em }}\right), \text { else }
\end{array}\right.
\end{aligned}
$$

where $e_{W_{e g r}}=W_{\text {egr }}^{s}-W_{\text {egr }}$. The controller parameters are automatically tuned by applying the method in Section 4 to the transient cycles in Table 1, changing the parameters in (27) to the parameters for the controllers in Figure 3, and setting the weighting factors to $\gamma_{\lambda_{O}}=1 / 8, \gamma_{\text {egr }}=$ $3 / 8$, and $\gamma_{l}=0$.

The full eighth order model, described in Section 1, is used as plant model to evaluate the four control systems above. All their control parameters are held constant for the entire operating region, i.e. no gain scheduling is used. In addition, a low pass filter is applied to all variables that are assumed to come from an observer. These variables are $\lambda_{\mathrm{O}}$, $x_{\text {egr }}, W_{\text {egr }}$, and $T_{e m}$.

Section 5.1-5.4 discuss various properties of the four controllers with the aid of steps in $\lambda_{\mathrm{O}}^{s}$ and $x_{\text {egr }}^{s}$. In all cases the operating point is $n_{e}=1200 \mathrm{rpm}$ and $u_{\delta}=130 \mathrm{mg} / \mathrm{cycle}$, that is not used in the four transient cycles in Table 1. Then, Section 5.5 compares and discusses the four controllers and their performance on all cycles defined by Table 1 . These cycles cover a large operating region and consist of large amplitudes in EGR and VGT position.

\subsection{Benefits with Integral Action}

In Figure 4, advantages with integral action are illustrated by comparing "CLF with integral action" and "CLF without integral action". Steps in $\lambda_{\mathrm{O}}^{s}$ and $x_{\text {egr }}^{s}$ are performed and the 

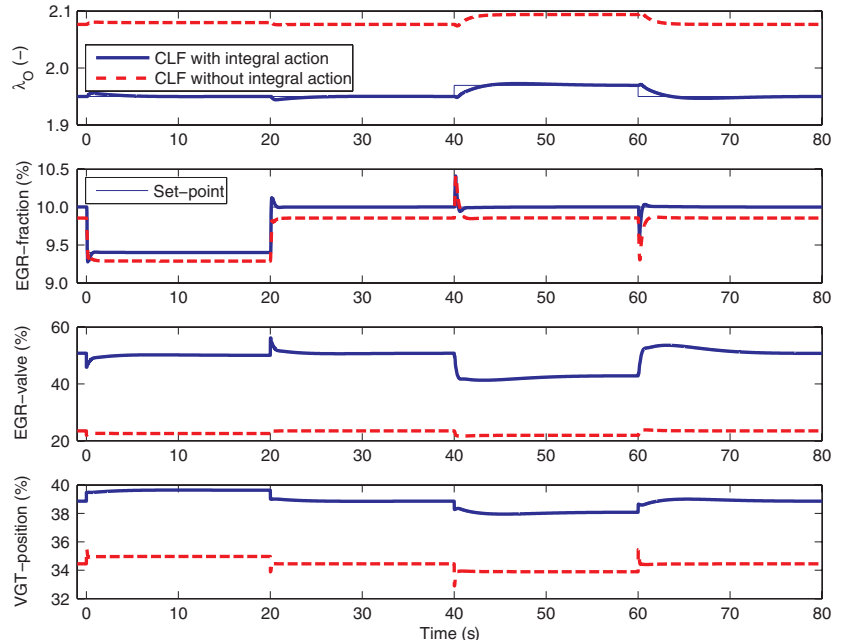

Figure 4

Illustrative example simulating CLF with and without integral action showing that the stationary control errors in $\lambda_{\mathrm{O}}$ and $x_{e g r}$ are reduced if an integral action is used.

result is that the stationary control errors in $\lambda_{O}$ and $x_{e g r}$ are reduced when the integral action is included.

\subsection{Benefits with Nonlinear Control and Input Transformation}

In Figures 5 and 6, advantages with nonlinear control and nonlinear input transformation are illustrated by comparing "CLF with integral action" and PID. In Figure 5 the same steps in $\lambda_{\mathrm{O}}^{s}$ and $x_{\text {egr }}^{s}$ are performed as in Figure 4. The result from Figure 5 is that PID gives slower control compared to CLF.

In Figure 6, "CLF with integral action" and PID are simulated at two other steps in $\lambda_{\mathrm{O}}^{s}$ and $x_{\text {egr }}^{s}$ compared to Figure 5. The operating point in Figure 6 renders higher DC-gains in $u_{\text {egr }} \rightarrow \lambda_{\mathrm{O}}$ and $u_{v g t} \rightarrow x_{\text {egr }}$ (that corresponds to the two loops that are used for feedback in Eq. 29, 30 compared to the operating point in Figure 5. The result is that PID gives oscillations, while CLF gives no oscillations. Since the same control parameters are used in Figures 5 and 6 for CLF respectively PID, the simulations show that the CLF based design handles the nonlinear effects in the Diesel engine, such as nonlinear gains. Figures 5 and 6 also show that the proposed design gives a stable system for the investigated operating points and achieves good control performance.

\subsection{Importance of the Nonlinear Input Transformation}

The previous section shows that the nonlinear control law (26) together with the nonlinear input transformation handle the nonlinear effects in the Diesel engine. The question now is: is it important to use both a nonlinear control law and a nonlinear input transformation or is it sufficient to use the PID control structure with a nonlinear input transformation in Figure 3 to handle the nonlinear effects? To answer this question "CLF with integral action" and "PID and input transformation" are compared in Figures 7 and 8 on the same steps in $\lambda_{\mathrm{O}}^{s}$ and $x_{\text {egr }}^{s}$ as were used in Figures 5 and 6 . Figures 7 and 8 show that there are only small differences in control performance between these two control systems. "PID and input transformation"
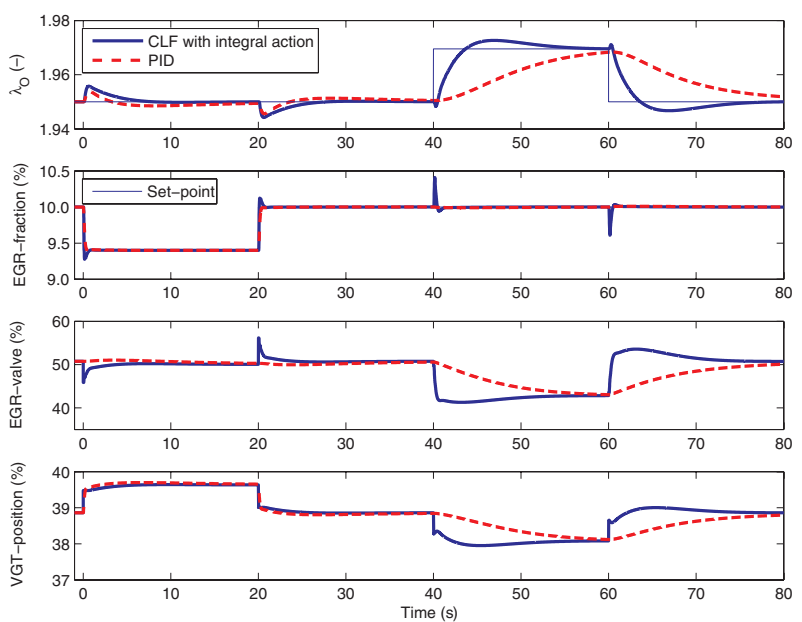

Figure 5

Illustrative example simulating "CLF with integral action" and PID showing that PID gives slower control compared to CLF.
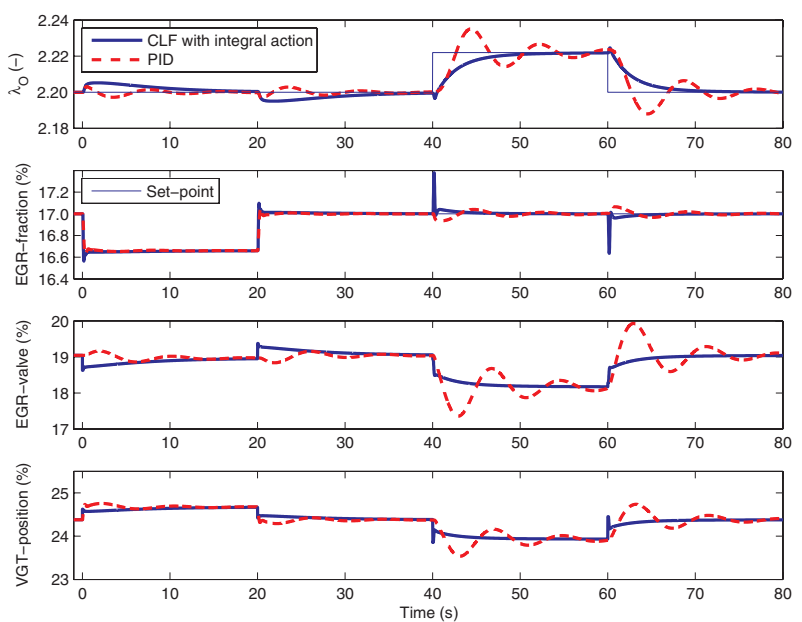

Figure 6

Illustrative example simulating "CLF with integral action" and PID at two other steps in $\lambda_{\mathrm{O}}^{s}$ and $x_{\text {egr }}^{s}$ compared to Figure 5 showing that PID gives oscillations and CLF gives no oscillations. 

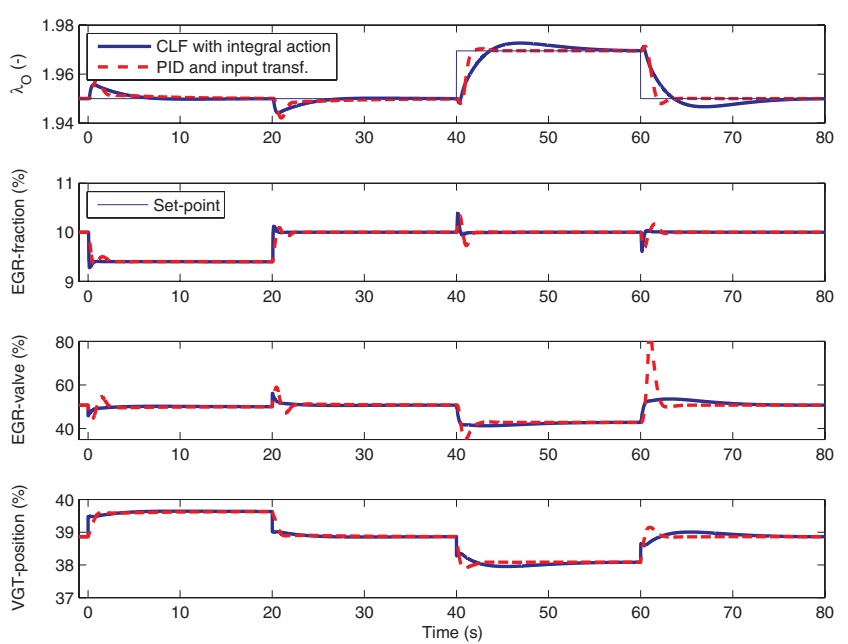

Figure 7

Illustrative example simulating "CLF with integral action" and "PID and input transformation" showing only small differences in control performance and that these control systems handle the nonlinear effects compared to PID in Figure 5.
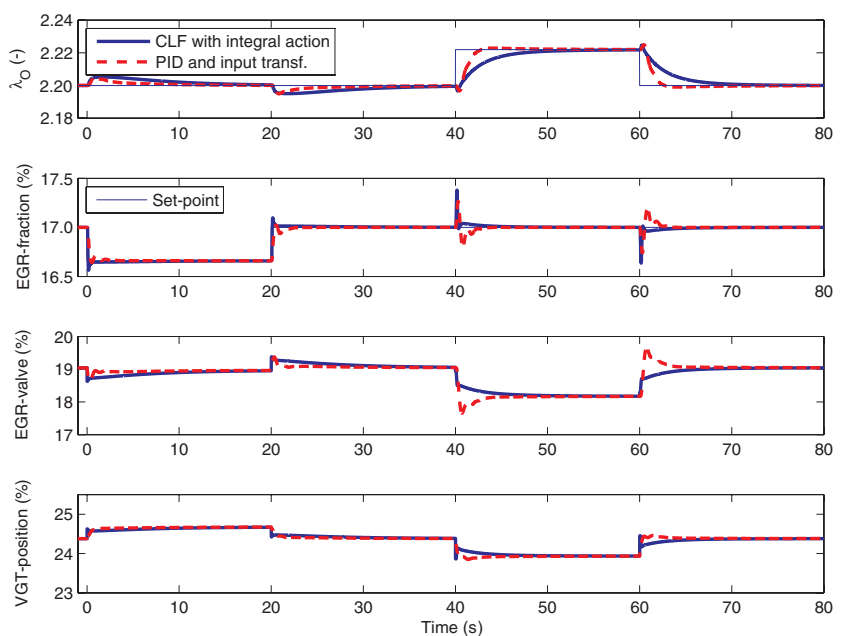

Figure 8

Illustrative example simulating "CLF with integral action" and "PID and input transformation" showing only small differences in control performance and that these control systems handle the nonlinear effects compared to PID in Figure 6.

gives a little faster response in $\lambda_{\mathrm{O}}$ while "CLF with integral action" gives a little smaller EGR-error. These differences are only due to that the tuning of the controllers have different trade-offs between $\lambda_{\mathrm{O}}$-error and $x_{\text {egr }}$-error. However, both these control systems handle the nonlinear effects in the Diesel engine. Consequently, it is important to use the input transformation to handle the nonlinear effects since both "PID and input transformation" and "CLF with integral action" use the input transformation. Further, it is sufficient to use the control structure in Figure 3 to handle the
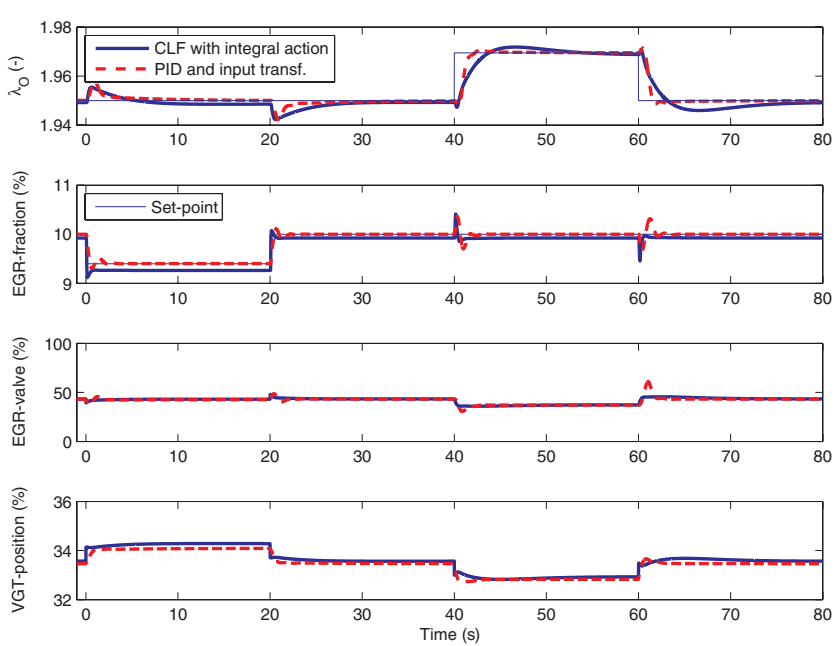

Figure 9

Illustrative example simulating "CLF with integral action" and "PID and input transformation". In this comparison, model errors are introduced in the EGR and turbine flow model for the simulation model showing that "CLF with integral action" gives control errors while "PID and input transformation" gives no control errors.
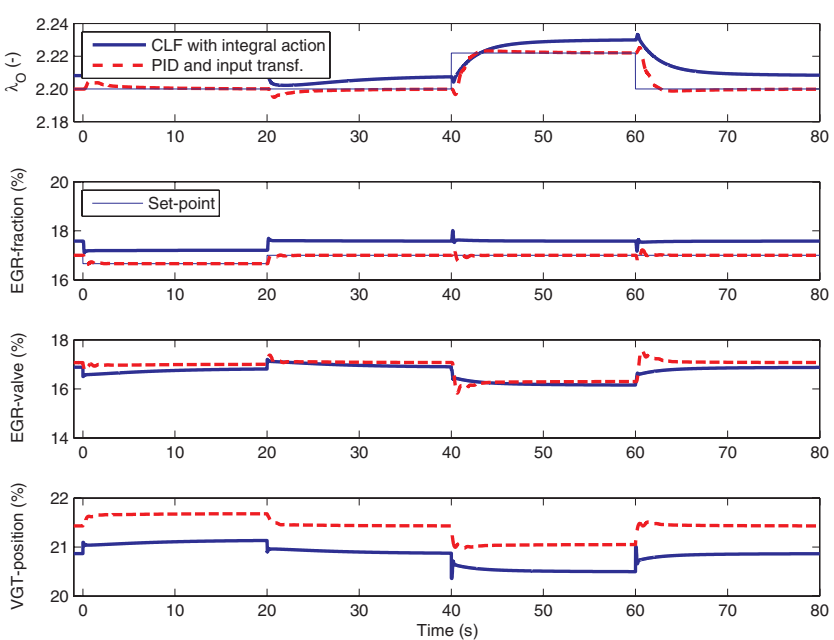

Figure 10

Illustrative example simulating "CLF with integral action" and "PID and input transformation". In this comparison, model errors are introduced in the EGR and turbine flow model for the simulation model showing that "CLF with integral action" gives control errors while "PID and input transformation" gives no control errors.

nonlinear effects since "PID and input transformation" and "CLF with integral action" have approximately the same control performance.

\subsection{Drawback with the Proposed CLF Based Control Design}

In Figures 9 and 10, disadvantages with the proposed control design are illustrated by comparing "CLF with integral 
action" and "PID and input transformation". In this comparison two model errors are introduced in the plant model. These errors are $10 \%$ positive errors in the maximum opening areas for the EGR and turbine flow model. These errors are selected to match the maximum model errors for the turbine and EGR flow that are in the order of $10-20 \%$ [15]. These introduced errors lead to that there are model errors in the input transformation, since the input transformation is developed by inverting the EGR and turbine flow model. The same steps in $\lambda_{\mathrm{O}}^{s}$ and $x_{\text {egr }}^{s}$ are investigated as in Figures 7 and 8 . The result is that "CLF with integral action" gives stationary control errors and "PID and input transformation" gives no stationary control errors. Consequently, "PID and input transformation" handles model errors in the input transformation while "CLF with integral action" is sensitive to these model errors. This is due to that "PID and input transformation" has integral parts in the PID controllers and "CLF with integral action" has no integral parts in the control law (26). The integral action in Figure 2 only handles model errors in the block "Set-point from 3rd order model" and the control law (26) only handles model errors in the block "CLF and inverse optimal control".

It is natural that the proposed control design in Figure 2 is sensitive to these model errors, since the input transformation is developed by using feedback linearization on the EGR and turbine flow model, i.e. by inverting these models. To handle these model errors, it is important to use integrators that directly affect the inputs to the input transformation according to the structure in Figure 3. Such integrators with feedback from $p_{e m}$ and/or $W_{\text {egr }}$ could be added to the structure in Figure 2 to handle these model errors. However, this has not been investigated.

\subsection{Comparison on the Four Transient Cycles}

The four control structures are compared on the four transient cycles defined in Table 1 by comparing $\lambda_{\mathrm{O}}$-error $E_{\lambda_{\mathrm{O}}}=\sum_{i=1}^{N} e_{\lambda_{\mathrm{O}}}^{2}\left(t_{i}\right)$ and $x_{\text {egr }}$-error $E_{x_{\text {egr }}}=\sum_{i=1}^{N} e_{x e g r}^{2}\left(t_{i}\right)$ where $t_{i}$ is the time at sample number $i$. Each control structure is simulated on two different simulation models. Simulation model A is the model in Section 1 and simulation model B is the same model except that the maximum opening areas are increased with $20 \%$ for the EGR and turbine flow models in the plant model. These model errors are larger than the model errors in Section 5.4 in order to get large effects on the control errors in Table 2. The model errors in Section 5.4 are set to $10 \%$ to avoid saturations in the actuators.

The goal is to investigate if the results in Section 5.1-5.4 are valid also for the four transient cycles. Table 2 shows that "CLF with integral action" reduces the errors compared to "CLF without integral action". Consequently, integral action reduces control errors and the result from Section 5.1 is valid. PID has higher errors than the other three controllers for simulation model A. Consequently, nonlinear
TABLE 2

The measures $E_{\lambda_{\mathrm{O}}}$ and $E_{x_{\text {egr }}}$ for four different controllers over the cycles defined in Table 1. Two different simulation models are used. Simulation model A is the model in Section 1 and simulation model B is the same model except that $20 \%$ positive model errors are introduced in the maximum opening areas for the EGR and turbine flow model. The measures are normalized with respect to PID for simulation model A.

\begin{tabular}{c||cc|cc}
\hline \multicolumn{1}{c||}{ Simulation model } & \multicolumn{2}{c|}{$\mathrm{A}$} & \multicolumn{2}{c}{$\mathrm{B}$} \\
Measure & $E_{\lambda_{\mathrm{O}}}$ & $E_{x_{\text {egr }}}$ & $E_{\lambda_{\mathrm{O}}}$ & $E_{x_{\text {egr }}}$ \\
\hline PID & 1.00 & 1.00 & 0.79 & 0.84 \\
PID and input transf. & 0.17 & 0.92 & 0.16 & 0.86 \\
CLF with integral action & 0.27 & 0.37 & 0.49 & 1.23 \\
CLF without integral action & 0.38 & 0.66 & 1.57 & 6.14 \\
\hline
\end{tabular}

control improves the control performance which confirms the result from Section 5.2. "PID and input transformation" and "CLF with integral action" have approximately the same control performance for simulation model A. The differences in $E_{\lambda_{\mathrm{O}}}$ and $E_{x_{e g r}}$ between these two controllers for simulation model $\mathrm{A}$ are only due to that the tuning of the controllers have different trade-offs between the $\lambda_{\mathrm{O}}$ and $x_{e g r}$-errors. Consequently, it is important to use the input transformation and it is sufficient to use "PID and input transformation" to reduce the control errors compared to PID which confirms the result from Section 5.3. Finally, "PID and input transformation" has lower errors compared to "CLF with integral action" for simulation model B which confirms the result from Section 5.4 that "PID and input transformation" handles model errors in the input transformation while "CLF with integral action" is sensitive to these model errors. This is also shown in Figures 11 and 12 that compare "CLF with integral action" and "PID and input transformation" for cycle 1 and 3 in Table 1 for simulation model B. Due to these results, "CLF with integral action" is not experimentally validated, instead "PID and input transformation" is recommended and experimentally validated in [19].

\section{CONCLUSIONS}

A nonlinear multivariable control design is proposed in [13] for control of EGR and VGT in Diesel engines. This design includes construction of a Lyapunov function, inverse optimal control, and a nonlinear input transformation which provides a control law that handles interactions and nonlinear properties in the system. This design is extended with integral action on the compressor mass flow to handle model errors so that the controller can track the performance variables specified in the outer loop. The design in [13] is locally asymptotic stable and robust, but it is shown that neither the here proposed design nor the one in [13] guarantee a globally asymptotically stable and robust system.

Comparisons between different control structures have been performed in simulations showing the following four 

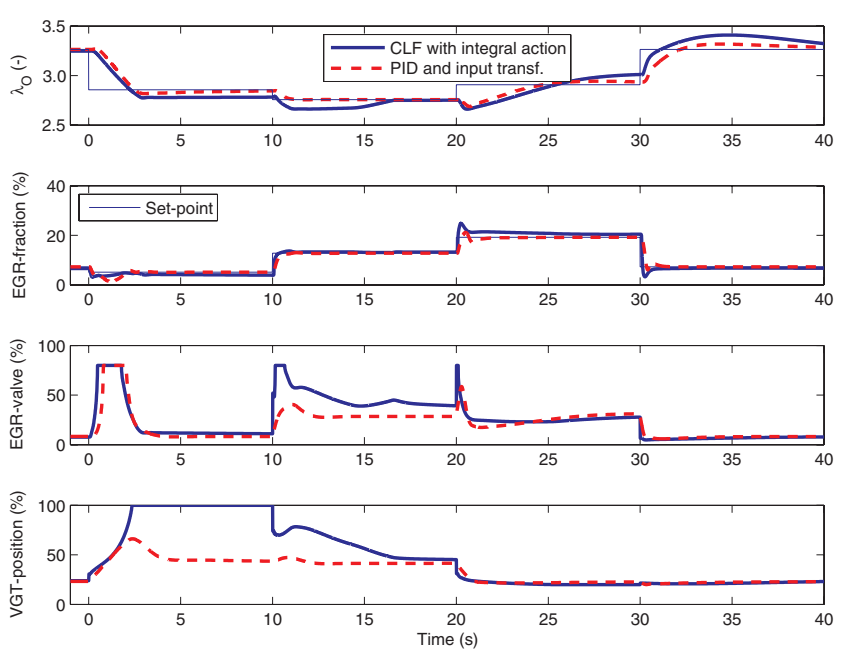

Figure 11

Illustrative example simulating "CLF with integral action" and "PID and input transformation" for cycle 1 in Table 1 . In this comparison, model errors are introduced in the EGR and turbine flow model for the simulation model showing that "CLF with integral action" gives control errors while "PID and input transformation" gives no control errors.
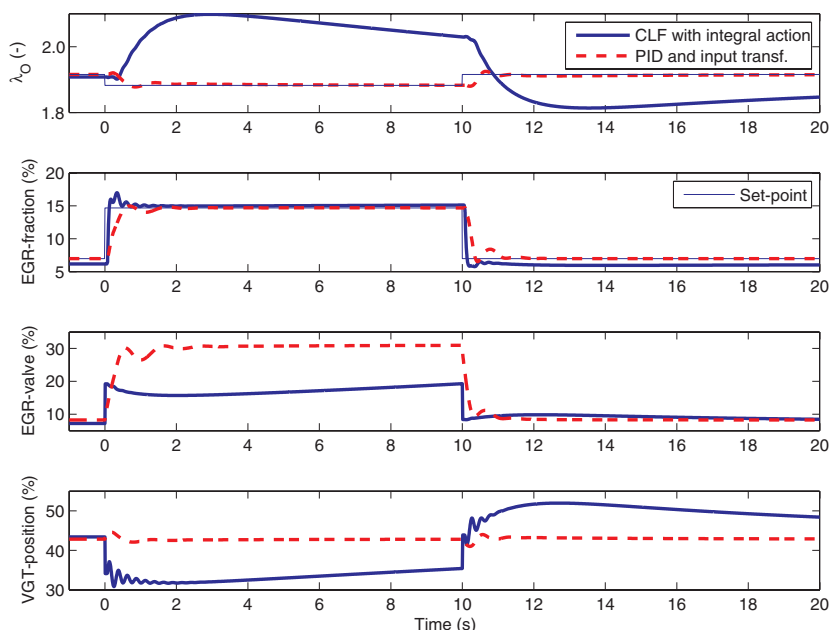

Figure 12

Illustrative example simulating "CLF with integral action" and "PID and input transformation" for cycle 3 in Table 1. In this comparison, model errors are introduced in the EGR and turbine flow model for the simulation model showing that "CLF with integral action" gives control errors while "PID and input transformation" gives no control errors.

points. Firstly, stationary control errors are reduced when integral action is used in the proposed design compared to a control design without integral action. Secondly, the proposed control design handles the nonlinear effects in the Diesel engine that results in less control errors compared to a control structure with PID controllers. Thirdly, it is important to use the input transformation and it is sufficient to use a control structure with PID controllers and input transformation to handle the nonlinear effects. Fourthly, the proposed control design is sensitive to model errors in the input transformation while a control structure with PID controllers and input transformation handle these model errors.

\section{APPENDIX}

\section{ANALYSIS OF STABILITY AND ROBUSTNESS PROPERTIES FOR THE PROPOSED DESIGN WITH INTEGRAL ACTION}

An analysis of the robustness properties for the proposed design with integral action is performed. This is done by checking the criteria (9) in the following proposition.

Proposition 1: For the system (17), the outputs (16), the CLF (25), and $R^{-1}=\operatorname{diag}\left\{\gamma_{1}, \gamma_{2}\right\}$ there exist $x=\left[\begin{array}{lll}y_{1} & y_{2} & p_{\text {im }} i\end{array}\right]^{T}$ such that $l(x)$ in $(8)$ is negative for any positive $\gamma_{1}, \gamma_{2}, c_{1}, c_{2}$, and $K$.

Proof: The function $l(x)$ becomes:

$$
l(x)=a_{1} y_{1}+a_{2} y_{2}+a_{3} y_{1} y_{2}+a_{4} y_{1}^{2}+a_{5} y_{2}^{2}
$$

where

$$
\begin{aligned}
a_{1}= & -2 c_{1}\left(-\frac{W_{c}}{\tau}-a\left(-k_{e} p_{\text {im }}+W_{c}+W_{e g r}^{s}\right)\right. \\
& \left.+b\left(W_{c}^{s}+W_{f}\right)\right) \\
a_{2}= & -2 c_{2}\left(\frac{d_{1}\left(\delta i+K y_{1}\right)}{d_{2}}-k_{e m}\left(-k_{e} p_{\text {im }}+W_{c}^{s}+W_{e g r}^{s}\right)\right) \\
a_{3}= & 2 a c_{1} c_{2} k_{e m} \gamma_{1}-2 b c_{1} c_{2} k_{e m} \gamma_{2} \\
a_{4}= & c_{1}^{2}\left(a^{2} \gamma_{1}+b^{2} \gamma_{2}\right)>0 \\
a_{5}= & c_{2}^{2} k_{e m}^{2}\left(\gamma_{1}+\gamma_{2}\right)>0
\end{aligned}
$$

Completing the squares in $l(x)$ gives:

$$
\begin{aligned}
l(x)= & a_{4}\left(y_{1}+\frac{a_{3}}{2 a_{4}} y_{2}+\frac{a_{1}}{2 a_{4}}\right)^{2} \\
& +b_{1}\left(y_{2}+\frac{b_{2}}{2 b_{1}}\right)^{2}-\frac{b_{2}^{2}}{4 b_{1}}-\frac{a_{1}^{2}}{4 a_{4}}
\end{aligned}
$$

where

$$
\begin{aligned}
& b_{1}=a_{5}-\frac{a_{3}^{2}}{4 a_{4}}=\frac{(a+b)^{2} c_{2}^{2} k_{e m}^{2} \gamma_{1} \gamma_{2}}{\gamma_{1} a^{2}+\gamma_{2} b^{2}}>0 \\
& b_{2}=a_{2}-\frac{a_{3} a_{1}}{2 a_{4}}
\end{aligned}
$$

There exist $x$ such that:

$$
\begin{aligned}
& y_{2}=-\frac{b_{2}}{2 b_{1}}, \quad y_{1}=-\frac{a_{3}}{2 a_{4}} y_{2}-\frac{a_{1}}{2 a_{4}} \\
& a_{1} \neq 0
\end{aligned}
$$


since $y_{1}$ and $y_{2}$ can be selected to satisfy (33) and $p_{\text {im }}$ can be selected to satisfy (34). For these $x$, it holds that:

$$
l(x)=-\frac{b_{2}^{2}}{4 b_{1}}-\frac{a_{1}^{2}}{4 a_{4}}<0
$$

for any positive $\gamma_{1}, \gamma_{2}, c_{1}, c_{2}$, and $K$.

Consequently, there exist no $\gamma_{1}, \gamma_{2}, c_{1}, c_{2}$, and $K$ such that $l(x)>0$ for all $x$ and therefore there is no guarantee that the control law (26) gives a globally robust system according to Section 2. $\dot{V}(x)$ is analyzed in the same way as the analysis of $l(x)$ above, showing that there exist no $\gamma_{1}, \gamma_{2}, c_{1}, c_{2}$, and $K$ such that $\dot{V}(x)<0$ for all $x$ and therefore there is no guarantee that the control law (26) gives a globally asymptotically stable system.

\section{ANALYSIS OF STABILITY AND ROBUSTNESS PROPERTIES FOR THE DESIGN WITHOUT INTEGRAL ACTION}

An analysis of the robustness properties for the design without integral action in [13] is performed. This is done by checking the criteria (9) in the same way as in Proposition 1 and this is done in the following proposition.

Proposition 2: For the design in [13], i.e. for the system (10), the outputs (12), the CLF:

$$
V=c_{1} y_{1}^{2}+c_{2} y_{2}^{2}+c_{3} z_{2}^{2}
$$

where $z_{2}=\left(p_{\text {im }} / p_{a m b}\right)^{\mu_{a}}-\left(p_{\text {im }}^{s} / p_{a m b}\right)^{\mu_{a}}$, and $R^{-1}=\operatorname{diag}\left\{\gamma_{1}, \gamma_{2}\right\}$ there exist $x=\left[\begin{array}{lll}y_{1} & y_{2} & z_{2}\end{array}\right]^{T}$ such that $l(x)$ in (8) is negative for any positive $\gamma_{1}, \gamma_{2}, c_{1}, c_{2}$, and $c_{3}$.

Proof: The function $l(x)$ becomes:

$$
\begin{aligned}
l(x)= & a_{1} y_{1}+a_{2} y_{2}+a_{3} y_{1} y_{2}+a_{4} y_{1}^{2}+a_{5} y_{2}^{2}+ \\
& a_{6} y_{1} z_{2}+a_{7} y_{2} z_{2}+a_{8} z_{2}^{2}+a_{9} z_{2}
\end{aligned}
$$

where

$$
\begin{aligned}
a_{1}= & -2 c_{1}\left(-\frac{W_{c}}{\tau}-a\left(-k_{e} p_{i m}+W_{c}+W_{e g r}^{s}\right)\right. \\
& \left.+b\left(W_{c}^{s}+W_{f}\right)\right) \\
a_{2}= & 2 c_{2} k_{e m}\left(W_{c}^{s}+W_{e g r}^{s}-k_{e} p_{i m}\right) \\
a_{3}= & 2 a c_{1} c_{2} k_{e m} \gamma_{1}-2 b c_{1} c_{2} k_{e m} \gamma_{2} \\
a_{4}= & c_{1}^{2}\left(a^{2} \gamma_{1}+b^{2} \gamma_{2}\right)>0 \\
a_{5}= & c_{2}^{2} k_{e m}^{2}\left(\gamma_{1}+\gamma_{2}\right)>0 \\
a_{6}= & -2 a c_{1} c_{3} k_{i m} \mu_{a} p_{a m b}^{-\mu_{a}} p_{i m}^{\mu_{a}-1} \gamma_{1}<0 \\
a_{7}= & -2 c_{2} c_{3} k_{e m} k_{i m} \mu_{a} p_{a m b}^{-\mu_{a}} p_{i m}^{\mu_{a}-1} \gamma_{1}<0 \\
a_{8}= & c_{3}^{2} k_{i m}^{2} \mu_{a}^{2} p_{a m b}^{-2 \mu_{a}} p_{i m}^{2 \mu_{a}-2} \gamma_{1}>0 \\
a_{9}= & -2 c_{3} k_{i m} \mu_{a} p_{a m b}^{-\mu_{a}} p_{i m}^{\mu_{a}-1}\left(-k_{e} p_{i m}+W_{c}+W_{e g r}^{s}\right)
\end{aligned}
$$

Completing the squares in $l(x)$ gives:

$$
\begin{aligned}
l(x)= & a_{4}\left(y_{1}+\frac{a_{3}}{2 a_{4}} y_{2}+\frac{a_{6}}{2 a_{4}} z_{2}+\frac{a_{1}}{2 a_{4}}\right)^{2} \\
& +b_{1}\left(y_{2}+\frac{b_{4}}{2 b_{1}} z_{2}+\frac{b_{2}}{2 b_{1}}\right)^{2}-\frac{b_{2}^{2}}{4 b_{1}}-\frac{a_{1}^{2}}{4 a_{4}}+b_{3} z_{2}
\end{aligned}
$$

where

$$
\begin{aligned}
b_{1}= & a_{5}-\frac{a_{3}^{2}}{4 a_{4}}=\frac{(a+b)^{2} c_{2}^{2} k_{e m}^{2} \gamma_{1} \gamma_{2}}{\gamma_{1} a^{2}+\gamma_{2} b^{2}}>0 \\
b_{2}= & a_{2}-\frac{a_{3} a_{1}}{2 a_{4}} \\
b_{4}= & a_{7}-\frac{a_{3} a_{6}}{2 a_{4}} \\
b_{3}= & a_{9}-\frac{a_{6} a_{1}}{2 a_{4}}-\frac{b_{2} b_{4}}{2 b_{1}}= \\
& -\frac{2 c_{3} k_{i m} \mu_{a}\left((b \tau-1) W_{c}+b \tau W_{f}\right)}{p_{a m b}^{\mu_{a}} p_{i m}^{1-\mu_{a}}(a+b) \tau}
\end{aligned}
$$

There exist $x$ such that:

$$
\begin{gathered}
y_{2}=-\frac{b_{4}}{2 b_{1}} z_{2}-\frac{b_{2}}{2 b_{1}}, y_{1}=-\frac{a_{3}}{2 a_{4}} y_{2}-\frac{a_{6}}{2 a_{4}} z_{2}-\frac{a_{1}}{2 a_{4}}(36) \\
b_{3} z_{2}<0
\end{gathered}
$$

since $y_{1}$ and $y_{2}$ can be selected to satisfy (36) and $z_{2}$ can be selected to satisfy (37). Note that $b_{3} \neq 0$ since $x \neq 0$ for these $x$. The $x$ that satisfy (36-37) gives:

$$
l(x)=-\frac{b_{2}^{2}}{4 b_{1}}-\frac{a_{1}^{2}}{4 a_{4}}+b_{3} z_{2}<0
$$

for any positive $\gamma_{1}, \gamma_{2}, c_{1}, c_{2}$, and $c_{3}$.

Consequently, there exist no $\gamma_{1}, \gamma_{2}, c_{1}, c_{2}$, and $c_{3}$ such that $l(x)>0$ for all $x$ and therefore there is no guarantee that the design in [13] gives a globally robust system according to Section 2. $\dot{V}(x)$ is analyzed in the same way as the analysis of $l(x)$ above, showing that there exist no $\gamma_{1}, \gamma_{2}, c_{1}, c_{2}$, and $c_{3}$ such that $\dot{V}(x)<0$ for all $x$ and therefore there is no guarantee that the design in [13] gives a globally asymptotically stable system. This property is stated without proof in [13].

\section{ACKNOWLEDGMENTS}

This research was supported by the VINNOVA Industry Excellence Center LINK-SIC and by the Linnaeus Center CADICS, funded by the Swedish Research Council.

\section{REFERENCES}

1 Heywood J.B. (1988) Internal Combustion Engine Fundamentals, McGraw-Hill Book Co.

2 Guzzella L., Amstutz A. (1998) Control of diesel engines, IEEE Contr. Syst. Mag. 18, 5, 53-71. 
3 Nieuwstadt M.J., Kolmanovsky I.V., Moraal P.E., Stefanopoulou A.G., Jankovic M. (2000) EGR-VGT control schemes: Experimental comparison for a high-speed diesel engine, IEEE Contr. Syst. Mag. 20, 3, 63-79.

4 Amstutz A., Del Re L. (1995) EGO sensor based robust output control of EGR in Diesel engines, IEEE Trans. Control Syst. Technol. 3, 39-48.

5 Nieuwstadt M., Moraal P.E., Kolmanovsky I.V., Stefanopoulou A., Wood P., Widdle M. (1998) Decentralized and multivariable designs for EGR-VGT control of a Diesel engine, in IFAC Workshop, Advances in Automotive Control, 1998.

6 Stefanopoulou A.G., Kolmanovsky I.V., Freudenberg J.S. (2000) Control of variable geometry turbocharged Diesel engines for reduced emissions, IEEE Trans. Contr. Syst. Technol. 8, 4, 733-745.

7 Ammann M., Fekete N.P., Guzzella L., Glattfelder A.H. (2003) Model-based Control of the VGT and EGR in a Turbocharged Common-Rail Diesel Engine: Theory and Passenger Car Implementation, SAE Technical paper 2003-01-0357.

8 Rückert J., Schloßer A., Rake H., Kinoo B., Krüger M., Pischinger S. (2001) Model based boost pressure and exhaust gas recirculation rate control for a diesel engine with variable turbine geometry, in IFAC Workshop: Advances in Automotive Control, 2001.

9 Chauvin J., Corde G., Petit N., Rouchon P. (2008) Motion planning for experimental airpath control of a diesel homogeneous charge-compression ignition engine, Control Eng. Pract. 16, 1081-1091.

10 Jung M., Glover K., Christen U. (2005) Comparison of uncertainty parameterisations for $\mathrm{H}$-infinity robust control of turbocharged diesel engines, Control Eng. Pract. 13, 15-25.

11 Rajamani R. (2005) Control of a variable-geometry turbocharged and wastegated diesel engine, Proc. IMechE Part D: J. Automobile Engineering 219, 11, 1361-1368.

12 Ferreau H.J., Ortner P., Langthaler P., del Re L., Diehl M. (2007) Predictive control of a real-world Diesel engine using an extended online active set strategy, Annu. Rev. Control.31, 2, 293-301.

13 Jankovic M., Jankovic M., Kolmanovsky I.V. (2000) Constructive lyapunov control design for turbocharged Diesel engines, IEEE Trans. Control Syst. Technol. 8, 2, 288-299.

14 Wahlström J., Eriksson L. (2008) Robust nonlinear EGR and VGT control with integral action for Diesel engines, in 17th IFAC World Congress, Seoul, Korea.

15 Wahlström J. (2009) Control of EGR and VGT for Emission Control and Pumping Work Minimization in Diesel Engines, PhD Thesis, Linköping University.

16 Sepulchre R., Jankovic M., Kokotovic P. (1997) Constructive Nonlinear Control, Springer-Verlag.

17 Jankovic M., Sepulchre R., Kokotovic P. (1999) CLF based designs with robustness to dynamic input uncertainties, Syst. Control Lett. 37, 45-54.

18 Bryson A.E., Yu-Chi Ho (1975) Applied Optimal Control, Taylor and Franchis.

19 Wahlström J., Eriksson L. (2010) Nonlinear input transformation for EGR and VGT control in Diesel engines, in $S A E$ 2010 Powertrains Fuels and Lubricants Meeting, SAE Technical paper 2010-01-2203, San Diego, USA.

20 Wahlström J., Eriksson L., Nielsen L. (2008) Controller tuning based on transient selection and optimization for a Diesel engine with EGR and VGT, in Electronic Engine Controls, SAE Technical paper 2008-01-0985, SP-2159, SAE World Congress, Detroit, USA.

21 Wahlström J., Eriksson L., Nielsen L. (2010) EGR-VGT control and tuning for pumping work minimization and emission control, IEEE Trans. Control Syst. Technol. 18, 4, 993-1003.

Final manuscript received in April 2011 Published online in October 2011 